Proceedings of the Institute of Mathematics and Mechanics,

National Academy of Sciences of Azerbaijan

Volume 46, Number 1, 2020, Pages 159-166

https://doi.org/10.29228/proc. 25

\title{
ON FREDHOLM PROPERTY OF A BOUNDARY VALUE PROBLEM FOR THIRD ORDER OPERATOR-DIFFERENTIAL EQUATIONS
}

\author{
SEADAT B. HEYDAROVA
}

\begin{abstract}
In the paper we obtain conditions of Fredholm property for third order operator-differential equations in a finite domain. All conditions were expressed by the coefficients of the given equation.
\end{abstract}

\section{Introduction}

Let $H$ be Hilbert space, $A$ be a positive definite self-adjoint operator in $H$. Obviously, the domain of definition of the operator $A^{\gamma}(\gamma \geq 0)-D\left(A^{\gamma}\right)$ becomes a Hilbert space $H_{\gamma}$ with respect to the scalar product $(x, y)_{\gamma}=\left(A^{\gamma} x, A^{\gamma} y\right)$. We consider $H_{0}=H$.

Denote by $L_{2}((0,1): H)$ a Hilbert space of functions $f(t)$ determined on the interval $(0,1)$ with the values in $H$, moreover

$$
\|f\|_{L_{2}((0 ; 1) ; H)}=\left(\int_{0}^{1}\|f(t)\|^{2} d t\right)^{\frac{1}{2}} .
$$

We introduce the Hilbert space

$$
W_{2}^{3}((0,1): H)=\left\{u, u^{\prime \prime \prime} \in L_{2}((0,1): H), A^{3} u \in L^{2}((0,1): H)\right\}
$$

with the norm

$$
\|u\|_{W_{2}^{3}((0,1): H)}=\left(\left\|u^{\prime \prime \prime}\right\|_{L_{2}((0,1): H)}^{2}+\left\|A^{3} u\right\|_{L_{2}((0,1: H))}^{2}\right)^{1 / 2}
$$

From the theorem on traces [3, Theorem 3.1] it follows that

$$
\stackrel{\circ}{W_{2}^{3}}((0,1): H)=\left\{u: u \in W_{2}^{3}((0,1): H), u(0)=0, u^{\prime}(1)=0, u^{\prime \prime}(1)=0\right\}
$$

is a complete subspace of the space $W_{2}^{3}((0,1): H)$.

Note that here and in the sequel, the derivatives are understood in the sense of distribution theory [3,p.14,15]. The space $W_{2}^{3}(R, H)$ is defined in the same way, where $R=(-\infty,+\infty)$.

From the theorem on intermediate derivatives and on traces [3, Theorem 2.3, Theorem 3.1], it follows that, if $u \in W_{2}^{3}((0,1): H)$, then $A^{3-k} u^{(k)} \in L_{2}((0,1)$ :

2010 Mathematics Subject Classification. 39B42, 46C05, 34K10.

Key words and phrases. Hilbert space, operator-differential equation, boundary-value problem, linear operator. 
$H), u^{(k)}(0) \in H_{3-k-\frac{1}{2}}, u^{(k)}(1) \in H_{3-k-\frac{1}{2}}(k=0,1,2)$ and $\left\|A^{3-k} u^{(k)}\right\|_{L_{2}((0,1): H)} \leq$ const $\|u\|_{W_{2}^{3}((0,1): H)}, \quad\left\|u^{(k)}(0)\right\|_{3-k-\frac{1}{2}} \leq$ const $\|u\|_{W_{2}^{3}((0,1): H)}, \quad\left\|u^{(k)}(1)\right\|_{3-k-\frac{1}{2}} \leq$ $\leq$ const $\|u\|_{W_{2}^{3}((0,1): H)}$.

It is known that $e^{-A t} \varphi \in W_{2}^{3}((0,1): H)$, if and only if $\varphi \in H_{\frac{5}{2}}$, where $e^{-A t}$ is a semigroup of bounded operators generated by the operator $(-A)$.

In the space $\stackrel{\circ}{W_{2}^{3}}((0,1): H)$ consider the following operator

$$
L u=u^{\prime \prime \prime}+A^{3} u+\sum_{j=0}^{2}\left(A_{3-j}+T_{3-j}\right) u^{(j)}
$$

where the coefficients of the operator $L$ satisfy the conditions:

(1) $A$ is a positive-definite self-adjoint operator with completely continuous inverse: $A^{-1}$

(2) The operators $B_{j}=A_{j} A^{-j}(j=1,2,3)$ are bounded in $H$;

(3) The operators $K_{j}=T_{j} A^{-j}(j=1,2,3)$ are completely continuous in $H$.

Obviously, from the theorem on intermediate derivatives it follows that, subject to the conditions 1$)-3), L$ is a bounded operator acting from $\stackrel{\circ}{W_{2}^{3}}((0,1): H)$ to $L_{2}((0,1): H)$.

In this paper, we find some sufficient conditions that provide Fredholm property of the operator $L$, i.e., $\operatorname{dim} \operatorname{Ker} L=\operatorname{codim} \operatorname{Im} L<\infty$ and $\operatorname{Im} L$ is a closed set in $L_{2}((0,1): H)$.

Then we apply this theorem on Fredholm solvability to the boundary value problem

$$
\begin{gathered}
u^{\prime \prime \prime}(t)+A^{3} u(t)+\sum_{j=0}^{2}\left(A_{3-j}+T_{3-j}\right) u^{(j)}(t)=f(t), t \in(0,1) \\
u(0)=0, u^{\prime}(1)=0, u^{\prime \prime}(1)=0 .
\end{gathered}
$$

Note that, unique solvability of the problem of type (1.1),(1.2) was studied in [8] for $A_{j}=T_{j}=0, j=1,2,3$ and for $T_{j}=0$ in $[1,2,4]$.

For second order operator-differential equations the unique solvability of the problem was considered for example in $[5,6,7]$.

\section{Some results}

In the space $\stackrel{\circ}{W_{2}^{3}}((0,1): H)$ we determine the operators

$$
P_{0} u=u^{\prime \prime \prime}+A^{3} u, P_{1} u=\sum_{j=0}^{2} A_{3-j} u^{(j)}, T u=\sum_{j=0}^{2} T_{3-j} u^{(j)} .
$$

The operator

$$
L u=P_{0} u+P_{1} u+T u
$$

from the space $\stackrel{\circ}{W_{2}^{3}}((0,1): H)$ to $L_{2}((0,1): H)$ is bounded. We have the following theorem. 
Theorem 2.1. The operator $P_{0}$ isomorphically maps the space $\stackrel{\circ}{W_{2}^{3}}((0,1): H)$ onto $L_{2}((0,1): H)$.

Proof. First we show that $\operatorname{Ker} P_{0}=\{0\}$. Indeed, for $u \in \stackrel{\circ}{W_{2}^{3}}((0,1): H)$ we have

$$
\begin{gathered}
\left\|P_{0} u\right\|_{L_{2}((0,1): H)}^{2}=\left\|u^{\prime \prime \prime}+A^{3} u\right\|_{L_{2}((0,1): H)}^{2}= \\
=\left\|u^{\prime \prime \prime}\right\|_{L_{2}((0,1): H)}^{2}+\left\|A^{3} u\right\|_{L_{2}((0,1): H)}^{2}+2 \operatorname{Re}\left(u^{\prime \prime \prime}, A^{3} u\right)_{L_{2}((0,1): H)} .
\end{gathered}
$$

Since for $u \in \stackrel{\circ}{W_{2}^{3}}((0,1): H)$ by integration by parts we get

$$
\left(u^{\prime \prime \prime}, A^{3} u\right)_{L_{2}((0,1): H)}=\left\|A^{3 / 2} u^{\prime}(0)\right\|^{2}-\left(A^{3} u, u^{\prime \prime \prime}\right)_{L_{2}((0,1): H)},
$$

we have $2 \operatorname{Re}\left(u^{\prime \prime \prime}, A^{3} u\right)_{L_{2}((0,1): H)}=\left\|A^{\frac{3}{2}} u^{\prime}(0)\right\|^{2}$. Then taking into account this equality in equality $(2.2)$, we have

$$
\mid P_{0} u\left\|_{L_{2}((0,1): H)}^{2}=\right\| u^{\prime \prime \prime}\left\|_{L_{2}((0,1): H)}^{2}+\right\| A^{3} u\left\|_{L_{2}((0,1): H)}^{2}+\right\| u^{\prime}(0) \|_{\frac{3}{2}}^{2} .
$$

From the condition $P_{0} u=0$ it follows that $u=0$, i.e. $\operatorname{Ker} P_{0}=\{0\}$. We now prove that $\operatorname{Im} P_{0}=L_{2}((0,1): H)$, i.e. the equation $P_{0} u=f$ has a solution for any $f \in L_{2}((0,1): H)$. Let $f_{1}(t)=f(t) t \in(0,1)$ and $f_{1}(t)=0$ for $R \backslash(0,1)$. Then $f_{1}(t) \in L_{2}(R: H)$ and $\left\|f_{1}\right\|_{L_{2}(R: H)}=\|f\|_{L_{2}((0,1): H)}$. It is easy to see that,

$$
u_{1}(t)=\frac{1}{\sqrt{2 \pi}} \int_{-\infty}^{+\infty}\left((i \xi)^{3}+A^{3}\right)^{-1} \hat{f}_{1}(\xi) e^{i \xi t} d \xi
$$

where $\hat{f}_{1}(\xi)$ is a Fourier transformation of the function $f_{1}(t)$ and satisfies the equation $u^{\prime \prime \prime}(t)+A^{3} u(t)=f_{1}(t)$ almost everywhere in $R=(-\infty, \infty)$. On the other hand, from the Plancherel theorem it follows that

$$
\begin{gathered}
\left\|u_{1}(t)\right\|_{W_{2}^{3}(R: H)}^{2}=\left\|u_{1}^{\prime \prime \prime}\right\|_{L_{2}((0,1): H)}^{2}+\left\|A^{3} u_{1}\right\|_{L_{2}(R: H)}^{2}=\left\|\xi^{3} \hat{u}_{1}(\xi)\right\|_{L_{2}(R: H)}^{2}+ \\
+\left\|A^{3} \hat{u}_{1}(\xi)\right\|_{L_{2}((0,1): H)}^{2}=\left\|\xi^{3}\left((i \xi)^{3}+A^{3}\right)^{-1} f_{1}(\xi)\right\|_{L_{2}(R)}^{2}+\left\|A^{3}\left((i \xi)^{3}+A^{3}\right)^{-1} \hat{f}_{1}(\xi)\right\|_{L_{2}(R)}^{2} \leq \\
\leq\left(\sup _{\xi \in R}\left\|\xi^{3}\left((i \xi)^{3}+A^{3}\right)^{-1}\right\|^{2}+\sup _{\xi \in R}\left\|A^{3}\left((i \xi)^{3}+A^{3}\right)^{-1}\right\|^{2}\right) \cdot\left\|\hat{f}_{1}(\xi)\right\| \|_{L_{2}(R ; H)}^{2}= \\
=\text { const }\|f\|_{L_{2}((0,1): H)}^{2} .
\end{gathered}
$$

Here we use the fact that if the operator $A$ is a self-adjoint positive-definite operator, then for any $\xi \in R$

$$
\left\|\xi^{3}\left((i \xi)^{3}+A^{3}\right)^{-1}\right\|=\sup _{\mu \in \sigma(A)}\left|\xi^{3}\left((i \xi)^{3}+\mu^{3}\right)^{-1}\right| \leq 1
$$

and

$$
\left\|A^{3}\left((i \xi)^{3}+A^{3}\right)^{-1}\right\|=\sup _{\mu \in \sigma(A)}\left|\mu^{3}\left((i \xi)^{3}+\mu^{3}\right)^{-1}\right| \leq 1 .
$$

Let us denote by $\alpha(t)=u_{1}(t)$ for $t \in[0,1]$ and $\alpha(t)=0$ for $t \in R \backslash[0,1]$.

Obviously, $\alpha(t)$ satisfies the equation $u^{\prime \prime \prime}(t)+A^{3} u(t)=f(t)$ almost everywhere in $(0,1)$ and $\alpha(t) \in W_{2}^{3}((0,1): H)$. Then by the theorem on traces, $\alpha^{(k)}(0) \in$ 
$H_{3-k-\frac{1}{2}}, \alpha^{(k)}(1) \in H_{3-k-\frac{1}{2}}(k=0,1,2)$. Now we will look for the solution of the equation $P_{0} u=f$ in the form

$$
u(t)=\alpha(t)+e^{-t A} x_{1}+e^{\omega(1-t) A} x_{2}+e^{\bar{\omega}(1-t) A} x_{3},
$$

where $x_{1}, x_{2}$ and $x_{3} \in H_{\frac{5}{2}}$ are unknown vectors, $\omega=-\frac{1}{2}-\frac{\sqrt{3}}{2} i$. From the condition $u \in \stackrel{\circ}{W_{2}^{3}}((0,1): H)$, it follows that

$$
x_{1}+e^{\omega A} x_{2}+e^{\bar{\omega} A} x_{3}=-\alpha(0), \quad e^{-A} x_{1}+\omega x_{2}+\bar{\omega} x_{3}=A^{-1} \alpha^{\prime}(1),
$$

$e^{-A} x_{1}+\omega^{2} x_{2}+\bar{\omega}^{2} x_{3}=-A^{-2} \alpha^{\prime \prime}(1)$.

Taking into account that the vectors $\alpha(0), A^{-1} \alpha^{\prime}(1),-A^{-2} \alpha^{\prime \prime}(1) \in H_{\frac{5}{2}}$, then solving this system we get $x_{1}, x_{2}$ and $x_{3} \in H_{\frac{5}{2}}$, i.e. $u(t) \in \stackrel{\circ}{W_{2}^{3}}((0,1): H)$ Consequently, $\operatorname{Im} P_{0}=L_{2}((0,1): H)$. The theorem is proved

From this theorem and theorem on intermediate derivatives it follows that the following norms

$$
N_{j}=\sup _{\substack{u \neq 0, \sim \\ u \in W_{2}^{3}((0,1): H)}}\left\|A^{3-j} u^{(j)}\right\| \cdot\left\|P_{0}^{-1} u\right\|^{-1} j=0,1,2 ;
$$

are finite since the norms $\|u\|_{W_{2}^{3}((0,1): H)}$ and $\left\|P_{0}^{-1} u\right\|_{L_{2}((0,1): H)}$ are equivalent in $\stackrel{\circ}{W_{2}^{3}}((0,1): H)$. Next we prove the following result

Theorem 2.2. We have the following estimations:

$$
N_{0} \leq 1 ; \quad N_{1} \leq 2^{\frac{1}{2}} \cdot 3^{-\frac{1}{4}}, \quad N_{2} \leq 2 \cdot 3^{-\frac{1}{2}} .
$$

Proof. From equality (2.3) it follows that $N_{0} \leq 1$. Prove that $N_{2} \leq 2 \cdot 3^{-\frac{1}{2}}$. Obviously, for $u \in \stackrel{\circ}{W_{2}^{3}}((0,1): H)\left(u(0)=0, u^{\prime}(1)=0, u^{\prime \prime}(1)=0\right)$.

$$
\begin{aligned}
\left\|A^{2} u^{\prime}\right\|_{L_{2}((0,1): H)}^{2} & =\left(A^{2} u^{\prime}, A^{2} u^{\prime}\right)_{L_{2}((0,1): H)}=-\left(A^{3} u, A u^{\prime \prime}\right)_{L_{2}((0,1): H)} \leq \\
& \leq\left\|A^{3} u\right\|_{L_{2}((0,1): H)} \cdot\left\|A u^{\prime \prime}\right\|_{L_{2}((0,1): H)} .
\end{aligned}
$$

Let $\varepsilon>0$ be any positive number. Then for any $u \in \stackrel{\circ}{W_{2}^{3}}((0,1): H)$ we have the equality

$$
\begin{gathered}
\left\|\varepsilon u^{\prime \prime \prime}+A u^{\prime \prime}+\frac{1}{\varepsilon} A u^{\prime}\right\|_{L_{2}((0,1): H)}^{2}=\varepsilon^{2}\left\|u^{\prime \prime \prime}\right\|_{L_{2}((0,1): H)}^{2}+\left\|A u^{\prime \prime}\right\|_{L_{2}((0,1): H)}^{2}+ \\
+\frac{1}{\varepsilon^{2}}\left\|A u^{\prime}\right\|_{L_{2}((0,1): H)}^{2}+2 \varepsilon \operatorname{Re}\left(u^{\prime \prime \prime}, A^{2} u^{\prime}\right)_{L_{2}((0,1): H)}+2 \operatorname{Re}\left(u^{\prime \prime \prime}, A u^{\prime}\right)_{L_{2}((0,1): H)}+ \\
+\frac{1}{\varepsilon} \cdot 2 \operatorname{Re}\left(A u^{\prime \prime}, A^{2} u^{\prime}\right)_{L_{2}((0,1): H)} .
\end{gathered}
$$

Integrating by parts, we have :

$$
\left(u^{\prime \prime \prime}, A u^{\prime \prime}\right)_{L_{2}((0,1): H)}=-\left\|u^{\prime \prime}(0)\right\|^{2}-\left(A u^{\prime \prime}, u^{\prime \prime \prime}\right)
$$

i.e.

$$
2 \varepsilon R e\left(u^{\prime \prime \prime}, A u^{\prime}\right)_{L_{2}((0,1): H)}=-\varepsilon\left\|u^{\prime \prime}(0)\right\|_{\frac{1}{2}}^{2} .
$$


In the same way we have

$$
2 \cdot \frac{1}{\varepsilon}\left(A u^{\prime \prime}, A^{2} u^{\prime}\right)_{L_{2}((0,1): H)}=-\frac{1}{\varepsilon}\left\|u^{\prime}(0)\right\|_{\frac{3}{2}}^{2} .
$$

Taking into account the equalities $(2.8),(2.9)$, from equality (2.7) we get:

$$
\begin{gathered}
\left\|\varepsilon u^{\prime \prime \prime}+A u^{\prime \prime}+\frac{1}{\varepsilon} A u^{\prime}\right\|_{L_{2}((0,1): H)}^{2}=\varepsilon^{2}\left\|u^{\prime \prime \prime}\right\|_{L_{2}((0,1): H)}^{2}+ \\
+\frac{1}{\varepsilon^{2}}\left\|A u^{\prime}\right\|_{L_{2}((0,1): H)}^{2}-\left\|A u^{\prime \prime}\right\|_{L_{2}((0,1): H)}^{2}-\left\|\varepsilon^{\frac{1}{2}} A^{\frac{1}{2}} u^{\prime \prime}(0)+\varepsilon^{-\frac{1}{2}} A^{\frac{3}{2}} u^{\prime}(0)\right\|^{2} .
\end{gathered}
$$

Hence we have

$$
\left\|A u^{\prime \prime}\right\|_{L_{2}((0,1): H)}^{2} \leq \varepsilon^{2}\left\|u^{\prime \prime \prime}\right\|_{L_{2}((0,1): H)}^{2}+\frac{1}{\varepsilon^{2}}\left\|A^{2} u^{\prime}\right\|_{L_{2}((0,1): H)}^{2} .
$$

Assuming $\varepsilon=\left\|A^{2} u^{\prime}\right\|_{L_{2}((0,1): H)}^{\frac{1}{2}} \cdot\left\|u^{\prime \prime \prime}\right\|_{L_{2}((0,1): H)}^{-\frac{1}{2}}$, we get

$$
\left\|A u^{\prime \prime}\right\|_{L_{2}((0,1): H)}^{2} \leq 2\left\|u^{\prime \prime \prime}\right\|_{L_{2}((0,1): H)}^{2} \cdot\left\|A^{2} u^{\prime}\right\|_{L_{2}((0,1): H)}^{2} .
$$

Now, substituting the inequality (2.6) in (2.10), we get

$$
\left\|A u^{\prime \prime}\right\|_{L_{2}((0,1): H)}^{2} \leq 2 \cdot\left\|u^{\prime \prime \prime}\right\|_{L_{2}((0,1): H)} \cdot\left\|A^{3} u\right\|_{L_{2}((0,1): H)}^{1 / 2} \cdot\left\|A u^{\prime \prime}\right\|_{L_{2}((0,1): H)}^{\frac{1}{2}}
$$

i.e.

$$
\left\|A u^{\prime \prime}\right\|_{L_{2}((0,1): H)} \leq 2^{\frac{2}{3}}\left\|A^{3} u\right\|_{L_{2}((0,1): H)}^{\frac{1}{3}} \cdot\left\|u^{\prime \prime \prime}\right\|_{L_{2}((0,1): H)}^{\frac{2}{3}} \cdot
$$

Then

$$
\begin{aligned}
\left\|A u^{\prime \prime}\right\|^{2} & \leq 2^{\frac{4}{3}} \cdot\left(\frac{1}{\eta^{2}}\left\|A^{3} u\right\|_{L_{2}((0,1): H)}^{2}\right)^{\frac{1}{3}} \cdot\left(\tau\left\|u^{\prime \prime \prime}\right\|_{L_{2}((0,1): H)}^{2}\right)^{\frac{2}{3}} \leq \\
& \leq 2^{\frac{4}{3}}\left(\frac{1}{3} \frac{1}{\eta^{2}}\left\|A^{3} u\right\|_{L_{2}((0,1): H)}^{2}+\frac{2}{3} \tau\left\|u^{\prime \prime \prime}\right\|_{L_{2}((0,1): H)}^{2}\right)
\end{aligned}
$$

for $\tau>0$.

Assuming $\eta=2^{-\frac{1}{3}}$, we have

$$
\left\|A u^{\prime \prime}\right\|_{L_{2}((0,1): H)}^{2} \leq \frac{4}{3}\|u\|_{W_{2}^{3}((0,1): H)}^{2} .
$$

Consequently,

$$
\left\|A u^{\prime \prime}\right\|_{L_{2}((0,1): H)} \leq 2 \cdot 3^{-\frac{1}{2}}\|u\|_{W_{2}^{3}((0,1): H)} .
$$

Now, substituting the inequality in (2.6), we get

$$
\left\|A^{2} u^{\prime}\right\|_{L_{2}((0,1): H)} \leq 2^{\frac{1}{2}} \cdot 3^{-\frac{1}{4}}\left\|P_{0} u\right\|_{L_{2}((0,1): H)} .
$$

i.e.

$$
N_{1} \leq 2^{\frac{1}{2}} \cdot 3^{-\frac{1}{4}} ; \quad N_{2} \leq 2 \cdot 3^{-\frac{1}{2}} .
$$

The Theorem is proved. 


\section{Main results}

We now prove the following theorem.

Theorem 3.1. Let conditions 1) and 2) be fulfilled, moreover the operator $B_{j}=A_{j} A^{-j}(j=1,2,3)$ satisfy the condition

$$
q=\alpha_{0}\left\|B_{3}\right\|+\alpha_{1}\left\|B_{2}\right\|+\alpha_{2}\left\|B_{1}\right\|<1,
$$

where $\alpha_{0}=1 \quad \alpha_{1}=2^{\frac{1}{2}} \cdot 3^{-\frac{1}{4}}, \alpha_{2}=2 \cdot 3^{-\frac{1}{2}}$. Then the operator $P_{0}+P_{1}$ isomorphically maps the space $\stackrel{\circ}{W}_{2}^{3}((0,1): H)$ onto $L_{2}((0,1): H)$.

Proof. Since $P_{0}$ is an isomorphism, after substitution of $P_{0} u=v$ we can write the equation $\left(P_{0}+P_{1}\right) u=f$ in the form $v+P_{1} P_{0}^{-1} v=f$. Since

$$
\begin{gathered}
\left\|P_{1} P_{0}^{-1} v\right\|_{L_{2}((0,1): H)}=\left\|P_{1} u\right\|_{L_{2}((0,1): H)} \leq \sum_{j=0}^{2}\left\|A_{3-j} u^{(j)}\right\|_{L_{2}((0,1): H)} \leq \\
\leq \sum_{j=0}^{2}\left\|A_{3-j} A^{-3+j}\right\|\left\|A^{3-j} u^{(j)}\right\|_{L_{2}((0,1): H)},
\end{gathered}
$$

taking into account the result of theorem 2.2 , we get

$$
\begin{aligned}
& \left\|P_{1} P_{0}^{-1} v\right\|_{L_{2}((0,1): H)} \leq \sum_{j=0}^{2}\left\|B_{3-j}\right\| \cdot N_{j} \cdot\left\|P_{0} u\right\|_{L_{2}((0,1): H)} \leq \\
& \quad \leq \sum_{j=0}^{2} \alpha_{j}\left\|B_{3-j}\right\| \cdot\|v\|_{L_{2}((0,1): H)}=q\|v\|_{L_{2}((0,1): H)} .
\end{aligned}
$$

Since $q<1$, the operator $E+P_{1} P_{0}^{-1}$ is invertible in the space $L_{2}((0,1): H)$. Then the operator $\left(P_{0}+P_{1}\right)$ is invertible in $L_{2}((0,1): H)$ and $\left(P_{0}+P_{1}\right)^{-1}=$ $P_{0}^{-1}\left(E+P_{1} P_{0}^{-1}\right)^{-1}$. Hence it follows that $\|u\|_{W_{2}^{3}((0,1): H)} \leq$ const $\|f\|_{L_{2}((0,1): H)}$. The theorem is proved.

Theorem 3.2. Let conditions 1) and 3) be fulfilled. Then the operator $T$ is a completely continuous operator from the space $W_{2}^{3}((0,1): H)$ to $L_{2}((0,1): H)$.

Proof. Since for $u \in \stackrel{\circ}{W_{2}^{3}}((0,1): H)$

$$
T u=T_{2} u^{\prime}+T_{1} u^{\prime \prime}+T_{3} u=K_{2} A^{2} u^{\prime}+K_{1} A u^{\prime \prime}+K_{0} A^{3} u,
$$

where $K_{0}, K_{1}$ and $K_{2}$ are completely continuous operators, then it suffices to prove that $K_{0} A^{3}, K_{2} A^{2} \frac{d}{d t}$ and $K_{1} A \frac{d^{2}}{d t^{2}}$ are completely continuous operators from $\stackrel{\circ}{W_{2}^{3}}((0,1): H)$ to $L_{2}((0,1): H)$. For definiteness we consider the operator $K_{1} A \frac{d^{2}}{d t^{2}}$. Since $K_{1}$ is a completely continuous operator, we can represent it in the form of a finite-dimensional operator and an operator with sufficiently small norm $K_{1}=\sum_{i=1}^{n}\left(\cdot, x_{i}\right) y_{i}+K_{1}^{\prime}$ : $\left\|K_{1}^{\prime}\right\|<\varepsilon$ for sufficiently small $\varepsilon>0$. Obviously, if $e_{k}(k=1,2, \ldots)$ is an orthonormal basis of the system of eigen-vectors of the 
operator $A$, (i.e. $A e_{k}=\lambda_{k} e_{k}$ ) then, $x_{i}=\sum_{p=1}^{n} c_{p_{i}} e_{i}+x_{i}(\varepsilon)$, moreover $\left\|x_{i}(\varepsilon)\right\| \leq \varepsilon$. Then for one - dimensional operator $K_{1, i}=\left(, e_{i}\right) y_{i}$ we have

$$
\left\|K_{1, i} A u^{\prime \prime}\right\|_{L_{2}((0,1): H)}=\lambda_{k}\left\|y_{i}\right\| \cdot\left|\left(u^{\prime \prime}(t), e_{k}\right)\right|_{L_{2}((0,1)} .
$$

Since for the function $\psi(t)=\left(u(t), e_{k}\right)$ we get

$$
\begin{aligned}
\left\|\psi^{\prime \prime}(t)\right\|_{L_{2}((0,1): H)}^{2}= & \int_{0}^{1}\left(\psi^{\prime \prime}(t), \bar{\psi}^{\prime \prime}(t)\right) d t=\left.\psi^{\prime}(t) \cdot \bar{\psi}^{\prime \prime}(t)\right|_{0} ^{1}-\left(\psi^{\prime}(t) \cdot \bar{\psi}^{\prime \prime \prime}(t)\right)_{L_{2}((0,1)}= \\
& =\left(\psi^{\prime}(t) \cdot \bar{\psi}^{\prime \prime \prime}(t)\right)_{L_{2}((0,1)} \leq\left\|\psi^{\prime}(t)\right\|\left\|\psi^{\prime \prime \prime}(t)\right\|
\end{aligned}
$$

and

$\|\psi(t)\|^{2}=\int_{0}^{1}\left(\psi^{\prime}(t), \bar{\psi}^{\prime}(t)\right) d t=\left.\psi(t) \cdot \psi^{\prime}(t)\right|_{0} ^{1}-\left(\psi(t), \psi^{\prime \prime}(t)\right)_{L_{2}} \leq\|\psi(t)\| \cdot\left\|\psi^{\prime \prime}(t)\right\|$, we obtain

i.e.

$$
\|\psi \prime(t)\|_{L_{2}((0,1): H)}^{2} \leq\|\psi(t)\| \cdot\left\|\psi^{\prime}(t)\right\|^{\frac{1}{2}} \cdot\left\|\psi^{\prime \prime \prime}(t)\right\|^{\frac{1}{2}}
$$

$$
\left\|\psi^{\prime}(t)\right\|_{L_{2}((0,1): H)} \leq\left\|\psi^{\prime \prime \prime}(t)\right\|^{\frac{1}{3}} \cdot\|\psi(t)\|^{\frac{2}{3}} \leq \varepsilon\left\|\psi^{\prime \prime \prime}(t)\right\|+M(\varepsilon)\|\psi(t)\|_{L_{2}((0,1)} .
$$

Thus,

$$
\left\|K_{1, i} A u^{\prime \prime}\right\|_{L_{2}((0,1): H)} \leq \varepsilon\|\psi\|_{W_{2}^{3}((0,1): H)}+M(\varepsilon)\|\psi\|_{L_{2}((0,1): H)} .
$$

Now, we can show that for any $\varepsilon>0$

$$
\left\|K_{1, i} A u^{\prime \prime}\right\|_{L_{2}((0,1): H)} \leq \varepsilon\|u\|_{W_{2}^{3}((0,1): H)}+M(\varepsilon)\|u\|_{L_{2}((0,1): H)} .
$$

Since the embedding $W_{2}^{3}((0,1): H) \subset L_{2}((0,1): H)$ is compact, $K_{1, i} A \frac{d^{2}}{d t^{2}}$ is a compact operator. Indeed, if $\left\{u_{k}\right\}_{k=1}^{\infty} \subset \stackrel{\circ}{W_{2}^{3}}((0,1): H)$ and $\left\|u_{k}\right\|_{W_{2}^{3}((0,1): H)} \leq M$, then there exists a sequence $u_{k, n} \subset\left\{u_{k}\right\}$, moreover, $u_{k, n} \rightarrow u_{0}$ in $L_{2}((0,1): H)$. Then from inequality 3.2 it follows that

$$
\left\|K_{1 i} A\left(u_{n}^{\prime \prime}-u_{m}^{\prime \prime}\right)\right\|_{L_{2}((0,1): H)} \leq 2 \varepsilon \cdot M+M(\varepsilon)\left\|u_{n}-u_{m}\right\|_{L_{2}((0,1): H)} .
$$

Since as $n, m \rightarrow \infty\left\|u_{n}-u_{m}\right\| \rightarrow 0$, and $\varepsilon$ is any positive number, we conclude that the sequence $\left\{K_{1, i} A u_{n}^{\prime \prime}\right\}_{n=1}^{\infty}$ converges in $L_{2}((0,1): H)$ i.e. the operator $K_{1, i}$ is a compact operator. Consequently, the operator $K_{1} A \frac{d^{2}}{d t^{2}}$ is a Fredholm operator from the space $\stackrel{\circ}{W_{2}^{3}}((0,1): H)$ to $L_{2}((0,1): H)$.

The theorem has been proved.

We now prove the basic theorem.

Theorem 3.3. Let conditions 1)-3) and (3.1) be fulfilled. Then $L: \stackrel{\circ}{W_{2}^{3}}((0,1)$ : $H) \rightarrow L_{2}((0,1): H)$ is a Fredholm operator.

Proof. Obviously, $L=P_{0}+P_{1}+T=\left(E+T\left(P_{0}+P_{1}\right)^{-1}\right)\left(P_{0}+P_{1}\right)$.

Since the operator $\left(P_{0}+P_{1}\right)$ is an isomorphism, $T\left(P_{0}+P_{1}\right)^{-1}$ is a compact operator, then $L$ is a Fredholm operator. 
Corollary 3.1. Let the conditions of theorem 3.3 be fulfilled. Then problem (1.1),(1.2) is Fredholm solvable.

\section{References}

[1] S. B. Heydarova, On a periodic type boundary value problem for a third order equation in Hilbert space, Trans. of National Academy of Sciences of Azerbaijan, Series of Physical-Technical and Mathematical Sciences 35(1) (2015), 42-46.

[2] S. B. Heydarova, On well defined solvability of a boundary value problem for a third order equation in Hilbert space, Izvestia Pedagogicheskovo Universiteta estestv. Nauki. (2) (2016) 52-60.

[3] J. L. Lions, E. Magenes, Inhomogeneous boundary value problems and their applications. M. Mir, 371 p. (1971), Russian.

[4] S. S. Mirzoyev, S. B. Heydarova, On a boundary-value problem for third order operator-differential equations on a finite interval Applied Mathematical Sciences 10 (11) (2016), 543-548, http://dx.doi.org/10.12988/ams.2016.512743.

[5] S. S. Mirzoev, M. D. Karaaslan, R. Z. Gumbataliev, To the theory of operatordifferential equations of the second order, Doklady Akademii Nauk 453 (6) (2013), 610-612.

[6] S.S.Mirzoyev, M.Yu.Salimov, On complete elementary solutions of a class of second order operator-differential equations, Sibirskii Matem. Zhurnal 51 (4) (2010), 815828, Russian.

[7] S.S. Mirzoyev, M.Yu. Salimov, On solvability of a boundary value problem for second order equation with operator coefficients in the boundary condition, Matem. zametki, 91(6) (2012), 861-864, Russian.

[8] E. Obrecht, Un problema ai limiti ben posto per lequazione differenziale $u^{\prime \prime \prime}=A u+f$ in uno spazio di Banach. Rendiconti del Seminario Matematico della Università di Padova 55 (1976), 243-274.

Seadat B. Heydarova

Institute of Mathematics and Mechanics NAS of Azerbaijan, Baku, Azerbaijan E-mail address: seadet1987heyderova@mail.ru

Received: November 20, 2019; Revised: March 12, 2020; Accepted: April 16, 2020 